\title{
Positive Solutions for a Second-Order $p$-Laplacian Boundary Value Problem with Impulsive Effects and Two Parameters
}

\author{
Meiqiang Feng \\ School of Applied Science, Beijing Information Science \& Technology University, Beijing, 100192, China \\ Correspondence should be addressed to Meiqiang Feng; meiqiangfeng@sina.com
}

Received 1 April 2014; Accepted 19 May 2014; Published 18 June 2014

Academic Editor: Patricia J. Y. Wong

Copyright (C) 2014 Meiqiang Feng. This is an open access article distributed under the Creative Commons Attribution License, which permits unrestricted use, distribution, and reproduction in any medium, provided the original work is properly cited.

The author considers an impulsive boundary value problem involving the one-dimensional $p$-Laplacian $-\left(\varphi_{p}\left(u^{\prime}\right)\right)^{\prime}=$ $\lambda \omega(t) f(t, u), 0<t<1, t \neq t_{k},\left.\Delta u\right|_{t=t_{k}}=\mu I_{k}\left(t_{k}, u\left(t_{k}\right)\right),\left.\Delta u^{\prime}\right|_{t=t_{k}}=0, k=1,2, \ldots, n, a u(0)-b u^{\prime}(0)=\int_{0}^{1} g(t) u(t) d t, u^{\prime}(1)=0$, where $\lambda>0$ and $\mu>0$ are two parameters. Using fixed point theories, several new and more general existence and multiplicity results are derived in terms of different values of $\lambda>0$ and $\mu>0$. The exact upper and lower bounds for these positive solutions are also given. Moreover, the approach to deal with the impulsive term is different from earlier approaches. In this paper, our results cover equations without impulsive effects and are compared with some recent results by Ding and Wang.

\section{Introduction}

Impulsive differential equations, which provide a natural description of observed evolution processes, are regarded as important mathematical tools for the better understanding of several real-world problems in applied sciences, such as population dynamics, ecology, biological systems, biotechnology, industrial robotics, pharmacokinetics, and optimal control. Therefore, the study of this class of impulsive differential equations has gained prominence, and it is a rapidly growing field. For the general theory of impulsive differential equations, we refer the reader to [1-3], whereas the applications of impulsive differential equations can be found in [4-20]. In particular, we would like to mention some results of Lin and Jiang [8] and Feng and Xie [10]. In [8], Lin and Jiang investigated the following Dirichlet boundary value problem with impulse effects:

$$
\begin{gathered}
-u^{\prime \prime}(t)=f(t, u(t)), \quad t \in J, t \neq t_{k}, \\
\left.\Delta u^{\prime}\right|_{t=t_{k}}=-I_{k}\left(u\left(t_{k}\right)\right), \quad k=1,2, \ldots, m, \\
u(0)=u(1)=0,
\end{gathered}
$$

and, by virtue of the fixed point index theory in cones, the authors obtained some sufficient conditions for the existence of multiple positive solutions.
Recently, using fixed point theorems in a cone, Feng and Xie [10] considered the existence of positive solutions for the following problem:

$$
\begin{gathered}
-u^{\prime \prime}(t)=f(t, u(t)), \quad t \in J, t \neq t_{k}, \\
-\left.\Delta u^{\prime}\right|_{t=t_{k}}=I_{k}\left(u\left(t_{k}\right)\right), \quad k=1,2, \ldots, n, \\
u(0)=\sum_{i=1}^{m-2} a_{i} u\left(\xi_{i}\right), \quad u(1)=\sum_{i=1}^{m-2} b_{i} u\left(\xi_{i}\right) .
\end{gathered}
$$

Moreover, differential equations with $p$-Laplacian arise naturally in the study of flow through porous media $p=$ $3 / 2$, nonlinear elasticity $p \geq 2$, glaciology $1 \leq p \leq 4 / 3$, and so forth. In recent years, many cases of the existence, multiplicity, and uniqueness of positive solution of differential equations with $p$-Laplacian have attracted considerable attention [21-46]. Here, it is worth mentioning the studies by Dai and Ma [25] and Kajikiya et al. [26]. In [25], Dai and Ma considered the following one-dimensional $p$-Laplacian problem:

$$
\begin{gathered}
\left(\varphi_{p}\left(u^{\prime}\right)\right)^{\prime}+f(t, u)=0, \quad t \in(0,1), \\
u(0)=u(1)=0 .
\end{gathered}
$$


By using the global bifurcation theory, the authors showed the existence of nodal solutions.

In [26], Kajikiya et al. investigated the following onedimensional $p$-Laplacian problem:

$$
\begin{aligned}
\left(\varphi_{p}\left(u^{\prime}\right)\right)^{\prime}+\lambda \omega(t) f(u) & =0, \quad t \in(0,1), \\
u(0)=u(1) & =0
\end{aligned}
$$

and, by virtue of the global bifurcation theory, they obtained the existence, nonexistence, uniqueness, and multiplicity of positive solutions as well as sign-changing solutions under suitable conditions imposed on the nonlinear term $f$.

At the same time, we notice that there has been a considerable attention on impulsive differential equations with one-dimensional $p$-Laplacian. For example, in [31], Ding and O'Regan studied the second-order $p$-Laplacian boundary value problems involving impulsive effects:

$$
\begin{gathered}
\left(\varphi_{p}\left(u^{\prime}(t)\right)\right)^{\prime}=-f(t, u(t)), \quad 0<t<1, t \neq t_{k} \\
\left.\Delta u\right|_{t=t_{k}}=I_{k}\left(u\left(t_{k}\right)\right), \\
\left.\Delta u^{\prime}\right|_{t=t_{k}}=0, \quad k=1,2, \ldots, m, \\
u(0)=u^{\prime}(1)=0,
\end{gathered}
$$

and, via Jensens inequality, the first eigenvalue of a relevant linear operator, and the Krasnoselskii-Zabreiko fixed point theorem, they obtained the existence and multiplicity of positive solutions under suitable conditions imposed on the nonlinear term $f$ and the impulsive terms $I_{k}$.

In [33], employing the classical fixed point index theorem for compact maps, Zhang and Ge obtained some sufficient conditions for the existence of multiple positive solutions of the following problem:

$$
\begin{gathered}
\left(\varphi_{p}\left(u^{\prime}(t)\right)\right)^{\prime}=-f(t, u(t)), \quad 0<t<1, t \neq t_{k}, \\
\left.\Delta u\right|_{t=t_{k}}=-I_{k}\left(u\left(t_{k}\right)\right), \\
\left.\Delta u^{\prime}\right|_{t=t_{k}}=0, \quad k=1,2, \ldots, m, \\
u(0)=\sum_{i=1}^{m-2} a_{i} u\left(\xi_{i}\right), \quad u^{\prime}(1)=0 .
\end{gathered}
$$

However, to the best of our knowledge, no paper has considered the second-order impulsive differential equations with one-dimensional $p$-Laplacian and two parameters till now; for example, see [4-20, 31, 32, 43-45] and the references therein. In this paper, we will use fixed point theorem to investigate the existence and multiplicity of positive solutions for a second-order impulsive differential equation involving one-dimensional $p$-Laplacian and two parameters.
Consider the following second-order impulsive differential equation with one-dimensional $p$-Laplacian:

$$
\begin{gathered}
-\left(\varphi_{p}\left(u^{\prime}\right)\right)^{\prime}=\lambda \omega(t) f(t, u), \quad 0<t<1, t \neq t_{k}, \\
\left.\Delta u\right|_{t=t_{k}}=\mu I_{k}\left(t_{k}, u\left(t_{k}\right)\right), \\
\left.\Delta u^{\prime}\right|_{t=t_{k}}=0, \quad k=1,2, \ldots, n, \\
a u(0)-b u^{\prime}(0)=\int_{0}^{1} g(t) u(t) d t, \quad u^{\prime}(1)=0,
\end{gathered}
$$

where $\lambda>0, \mu>0$ are two parameters, $\varphi_{p}(s)=|s|^{p-2} s, p>1$, $\left(\varphi_{p}\right)^{-1}=\varphi_{q},(1 / p)+(1 / q)=1, a, b>0, \omega$ may be singular at $t=0$ and/or $t=1, t_{k}(k=1,2, \ldots, n$, where $n$ is fixed positive integer) are fixed points with $0<t_{1}<t_{2}<\cdots<t_{k}<\cdots<$ $t_{n}<1$, and $\left.\Delta u\right|_{t=t_{k}}$ denotes the jump of $u(t)$ at $t=t_{k}$; that is,

$$
\left.\Delta u\right|_{t=t_{k}}=u\left(t_{k}^{+}\right)-u\left(t_{k}^{-}\right),
$$

where $u\left(t_{k}^{+}\right)$and $u\left(t_{k}^{-}\right)$represent the right-hand limit and lefthand limit of $u(t)$ at $t=t_{k}$, respectively. In addition, $\omega, f, I_{k}$, and $g$ satisfy the following:

$\left(H_{1}\right) \omega$ is a nonnegative measurable function on $(0,1)$ and $\omega \neq \equiv$ on any open subinterval in $(0,1)$;

$\left(H_{2}\right) f \in C([0,1] \times[0,+\infty),[0,+\infty))$ with $f(t, u)>0$ for all $t$ and $u>0$;

$\left(H_{3}\right) I_{k} \in C([0,1] \times[0,+\infty),[0,+\infty))$ with $I_{k}(t, u)>0$ $(k=1,2, \ldots, n)$ for all $t$ and $u>0$;

$\left(H_{4}\right) g \in L^{1}[0,1]$ is nonnegative and $\sigma \in[0, a)$, where

$$
\sigma=\int_{0}^{1} g(t) d t
$$

Some special cases of (7) have been investigated. For example, Ding and Wang [14] considered problem (7) with $p=2, \lambda=1, \mu=1$, and $\omega(t) \equiv 1, t \in[0,1]$. By using Krasnoselskii's fixed point theorem, they proved the existence results of positive solutions of problem (7). However, the authors only obtained that problem (7) has at least one positive solution.

Motivated by the papers mentioned above, we will extend the results of $[11,14,23,31,33,47,48]$ to problem (7). We remark that on impulsive differential equations with a parameter only a few results have been obtained, not to mention impulsive differential equations with two parameters; see, for instance, $[12,18,19,45]$. These results only dealt with the case that $p=2$ and $\mu=1$. Many difficulties occur when we study problem (7); for example, it is difficult to construct the cone and the operator because its state is discontinuous. It is also difficult to deal with $\lambda$ and $\mu$ because of $\lambda$ with onedimensional $p$-Laplacian, and $\mu$ without one-dimensional $p$ Laplacian in the same equation (21). In this paper, we try to solve this kind of problem. Moreover, we will use a different approach to deal with the impulsive term to obtain the existence and multiplicity of positive solutions for problem (7); for details, see the proof of Theorem 1. 
The rest of the paper is organized as follows: in Section 2, we state the main results of problem (7). In Section 3, we provide some preliminary results, and the proofs of the main results together with several technical lemmas are given in Section 4 . The final section of the paper contains an example to illustrate the theoretical results.

\section{Main Results}

In this section, we state the main results, including existence and multiplicity results of positive solutions for problem (7).

For convenience, we introduce the following notations:

$$
\begin{gathered}
f^{0}=\limsup _{u \rightarrow 0^{+}} \max _{t \in J} \frac{f(t, u)}{\varphi_{p}(u)}, \quad f^{\infty}=\limsup _{u \rightarrow \infty} \max _{t \in J} \frac{f(t, u)}{\varphi_{p}(u)}, \\
f_{0}=\liminf _{u \rightarrow 0^{+}} \min _{t \in J} \frac{f(t, u)}{\varphi_{p}(u)}, \quad f_{\infty}=\liminf _{u \rightarrow \infty} \min _{t \in J} \frac{f(t, u)}{\varphi_{p}(u)}, \\
I^{0}(k)=\limsup _{u \rightarrow 0^{+}} \max _{t \in J} \frac{I_{k}(t, u)}{u}, \\
I^{\infty}(k)=\limsup _{u \rightarrow \infty} \max _{t \in J} \frac{I_{k}(t, u)}{u}, \\
I_{0}(k)=\liminf _{u \rightarrow 0^{+}} \min _{t \in J} \frac{I_{k}(t, u)}{u}, \\
I_{\infty}(k)=\liminf _{u \rightarrow \infty} \min _{t \in J} \frac{I_{k}(t, u)}{u}, \\
J=[0,1], \quad k=1,2, \ldots, n .
\end{gathered}
$$

Moreover, we choose four numbers $r, r_{1}, r_{2}$, and $R$ satisfying

$$
0<r<r_{1}<\delta r_{2}<r_{2}<R<+\infty
$$

where $\delta$ is defined in (23).

Theorem 1. Assume that $\left(H_{1}\right)-\left(H_{4}\right)$ hold and $f_{\infty}, f^{\infty}, I_{\infty}(k)$, and $I^{\infty}(k)(k=1,2, \ldots, n)$ are positive constants. Then,

(i) there exist $\lambda_{0}>0$ and $\mu_{0}>0$ such that, for any $\lambda>\lambda_{0}$ and $\mu>\mu_{0}$, problem (7) has a positive solution $u$ with

$$
\delta r \leq u(t) \leq \frac{1}{\delta} R, \quad t \in J
$$

(ii) there exist $\bar{\lambda}_{0}>0$ and $\bar{\mu}_{0}>0$ such that, for any $0<\lambda<$ $\bar{\lambda}_{0}$ and $0<\mu<\bar{\mu}_{0}$, problem (7) has a positive solution $u$ with property (12).

Theorem 2. Assume that $\left(H_{1}\right)-\left(H_{4}\right)$ hold and $f_{0}, f^{0}, I_{0}(k)$, and $I^{0}(k)(k=1,2, \ldots, n)$ are positive constants. Then,

(i) there exist $\lambda_{0}>0$ and $\mu_{0}>0$ such that, for any $\lambda>\lambda_{0}$ and $\mu>\mu_{0}$, problem (7) has a positive solution $u$ with

$$
\delta r \leq u(t) \leq R, \quad t \in J
$$

(ii) there exist $\bar{\lambda}_{0}>0$ and $\bar{\mu}_{0}>0$ such that, for any $0<\lambda<$ $\bar{\lambda}_{0}$ and $0<\mu<\bar{\mu}_{0}$, problem (7) has a positive solution $u$ with property (13).

Remark 3. Some ideas of the proof of Theorems 1 and 2 are from Yan [47]. In [47], Yan studied a class of the periodic impulsive functional differential equations with two parameters and proved the following existence result by using a well-known fixed point index theorem due to Krasnoselskii.

Theorem 4 (see [47, Theorem 3.1]). Assume that $\left(A_{1}\right)-\left(A_{6}\right)$ hold and $f^{0}, f_{\infty}, I_{\infty}$, and $I^{0}$ are positive constants. If

$$
\begin{gathered}
\beta\left(\lambda f^{0} P+\mu I^{0} Q\right)<1, \\
\alpha \sigma\left(\lambda f_{\infty} P+\mu I_{\infty} Q\right)>1,
\end{gathered}
$$

then problem (7) has a positive $\omega$-periodic solution.

It is not difficult to see that the conditions of Theorem 4 are not the optimal conditions which guarantee the existence of at least one positive $\omega$-periodic solution for the related problem. In fact, if

$$
\beta\left(\lambda f^{0} P+\mu I^{0} Q\right)<1
$$

or

$$
\alpha \sigma\left(\lambda f_{\infty} P+\mu I_{\infty} Q\right)>1
$$

we can prove that the problem studied in [47] has at least one positive $\omega$-periodic solution, respectively.

Theorem 5. Assume that $\left(H_{1}\right)-\left(H_{4}\right)$ hold.

(i) If $f^{\infty}=0$ and $I^{\infty}(k)=0, k=1,2, \ldots, n$, then there exist $\lambda_{0}>0$ and $\mu_{0}>0$ such that, for any $\lambda>\lambda_{0}$ and $\mu>\mu_{0}$, problem (7) has a positive solution $u$ with property (12).

(ii) If $f^{0}=0$ and $I^{0}(k)=0, k=1,2, \ldots, n$, then there exist $\lambda_{0}>0$ and $\mu_{0}>0$ such that, for any $\lambda>\lambda_{0}$ and $\mu>\mu_{0}$, problem (7) has a positive solution $u$ with property (13).

(iii) If $f^{0}=f^{\infty}=I^{\infty}(k)=I^{0}(k)=0, k=1,2, \ldots, n$, then there exist $\lambda_{0}>0$ and $\mu_{0}>0$ such that, for any $\lambda>\lambda_{0}$ and $\mu>\mu_{0}$, problem (7) has at least two positive solutions $u_{1}$ and $u_{2}$ with

$$
\delta r \leq u_{1}(t) \leq r_{1}<\delta r_{2} \leq u_{2}(t) \leq R, \quad t \in J .
$$

Theorem 6. Assume that $\left(H_{1}\right)-\left(H_{4}\right)$ hold.

(i) If $f_{\infty}=\infty$ and $I_{\infty}(k)=\infty, k=1,2, \ldots, n$, then there exist $\bar{\lambda}_{0}>0$ and $\bar{\mu}_{0}>0$ such that, for any $0<\lambda<\bar{\lambda}_{0}$ and $0<\mu<\bar{\mu}_{0}$, problem (7) has a positive solution $u$ with property (12).

(ii) If $f_{0}=\infty$ and $I_{0}(k)=\infty, k=1,2, \ldots, n$, then there exist $\bar{\lambda}_{0}>0$ and $\bar{\mu}_{0}>0$ such that, for any $0<\lambda<\bar{\lambda}_{0}$ and $0<\mu<\bar{\mu}_{0}$, problem (7) has a positive solution $u$ with property (13). 
(iii) If $f_{0}=f_{\infty}=I_{\infty}(k)=I_{0}(k)=+\infty, k=1,2, \ldots, n$, then there exist $\bar{\lambda}_{0}>0$ and $\bar{\mu}_{0}>0$ such that, for any $0<\lambda<\bar{\lambda}_{0}$ and $0<\mu<\bar{\mu}_{0}$, problem (7) has at least two positive solutions $u_{1}$ and $u_{2}$ with

$$
\delta r \leq u_{1}(t) \leq r_{1}<\delta r_{2} \leq u_{2}(t) \leq \frac{1}{\delta} R, \quad t \in J .
$$

Remark 7. Some ideas of the proof of Theorems 5 and 6 are from Graef et al. [48].

\section{Preliminaries}

Let $J^{\prime}=J \backslash\left\{t_{1}, t_{2}, \ldots, t_{n}\right\}$ and let $E$ be the Banach space:

$E=\left\{u \mid u: J \longrightarrow \mathbb{R}\right.$ is continuous at $t \neq t_{k}$,

$$
\left.u\left(t_{k}^{-}\right)=u\left(t_{k}\right), u\left(t_{k}^{+}\right) \text {exist, } k=1,2, \ldots, n\right\}
$$

with $\|u\|=\max _{0 \leq t \leq 1}|u(t)|$. We denote

$$
\Omega_{r}:=\{u \in E:\|u\|<r\}
$$

for all $r>0$ in the sequel.

In our main results, we will make use of the following definitions and lemmas.

Definition 8 (see [49]). Let $E$ be a real Banach space over $\mathbb{R}$. A nonempty closed set $P \subset E$ is said to be a cone provided that

(i) $a u+b v \in P$ for all $u, v \in P$ and all $a \geq 0, b \geq 0$;

(ii) $u,-u \in P$ implies $u=0$.

Every cone $P \subset E$ induces an ordering in $E$ given by $x \leq y$ if and only if $y-x \in P$.

Definition 9. A function $u \in E \cap C^{1}(0,1)$ with $\varphi_{p}\left(u^{\prime}\right) \in$ $C^{1}(0,1)$ is called a solution of (7) if it satisfies (7). If $u(t) \geq 0$ and $u(t) \neq \equiv 0$ on $J$, then $u$ is called a positive solution of (7).

Lemma 10. Assume that $\left(H_{1}\right)-\left(H_{4}\right)$ hold. Then, $u \in E \cap$ $C^{1}(0,1)$ with $\varphi_{p}\left(u^{\prime}\right) \in C^{1}(0,1)$ is a solution of problem (7) if and only if $u \in E$ is a solution of the following impulsive integral equation:

$$
\begin{aligned}
u(t)=\int_{0}^{t} \varphi_{q}\left(\lambda \int_{s}^{1} \omega(r) f(r, u(r)) d r\right) d s & \\
& +\mu \sum_{t_{k}<t} I_{k}\left(t_{k}, u\left(t_{k}\right)\right)+\frac{1}{a-\sigma} \\
& \times\left\{\int_{0}^{1} g(t)\left[\int_{0}^{t} \varphi_{q}\left(\lambda \int_{s}^{1} \omega(r) f(r, u(r)) d r\right) d s\right] d t\right. \\
& +b \varphi_{q}\left(\lambda \int_{0}^{1} \omega(s) f(s, u(s)) d s\right) \\
& \left.+\mu \int_{0}^{1} g(t) \sum_{t_{k}<t} I_{k}\left(t_{k}, u\left(t_{k}\right)\right) d t\right\} .
\end{aligned}
$$

Moreover, if $u$ is a positive solution of problem (7), then

$$
\min _{t \in J} u(t) \geq \delta\|u\|
$$

where

$$
\delta=\frac{\int_{0}^{1} \operatorname{tg}(t) d t}{a-\int_{0}^{1} g(t) d t+\int_{0}^{1} \operatorname{tg}(t) d t} .
$$

Proof. First, suppose that $u \in E$ is a solution of problem (7). It is easy to see by integration of (7) that

$$
-\phi_{p}\left(u^{\prime}(1)\right)+\phi_{p}\left(u^{\prime}(t)\right)=\int_{t}^{1} \lambda \omega(s) f(s, u(s)) d s .
$$

By the boundary condition $u^{\prime}(1)=0$, we have

$$
\begin{aligned}
& u^{\prime}(t)=\phi_{q}\left(\int_{t}^{1} \lambda \omega(s) f(s, u(s)) d s\right), \\
& u^{\prime}(0)=\phi_{q}\left(\int_{0}^{1} \lambda \omega(s) f(s, u(s)) d s\right) .
\end{aligned}
$$

If $0<t \leq t_{1}$, integrating (25) from 0 to $t$ we obtain

$$
u(t)-u(0)=\int_{0}^{t} \phi_{q}\left(\int_{s}^{1} \lambda \omega(r) f(r, u(r)) d r\right) d s .
$$

If $t_{1}<t \leq t_{2}$, integrating (25) from 0 to $t_{1}$ we obtain

$$
u\left(t_{1}\right)-u(0)=\int_{0}^{t_{1}} \phi_{q}\left(\int_{s}^{1} \lambda \omega(r) f(r, u(r)) d r\right) d s,
$$

and integrating (25) from $t_{1}$ to $t$ we obtain

$$
u(t)-u\left(t_{1}^{+}\right)=\int_{t_{1}}^{t} \phi_{q}\left(\int_{s}^{1} \lambda \omega(r) f(r, u(r)) d r\right) d s .
$$

It follows that

$$
\begin{aligned}
u(t)-u(0)= & \int_{0}^{t} \phi_{q}\left(\int_{s}^{1} \lambda \omega(r) f(r, u(r)) d r\right) d s \\
& +\mu I_{1}\left(t_{1}, u\left(t_{1}\right)\right), \quad t_{1}<t \leq t_{2} .
\end{aligned}
$$

For $t_{k}<t \leq t_{k+1}$, repeating the process we have

$$
\begin{aligned}
u(t)= & u(0)+\int_{0}^{t} \phi_{q}\left(\int_{s}^{1} \lambda \omega(r) f(r, u(r)) d r\right) d s \\
& +\mu \sum_{t_{k}<t} I_{k}\left(t_{k}, u\left(t_{k}\right)\right) .
\end{aligned}
$$

Combining this with the boundary condition, we have

$$
\begin{aligned}
u(0)= & \frac{1}{a-\int_{0}^{1} g(t) d t} \\
& \times\left\{\int_{0}^{1} g(t)\left[\int_{0}^{t} \phi_{q}\left(\int_{s}^{1} \lambda \omega(r) f(r, u(r)) d r\right) d s\right] d t\right. \\
& +b \phi_{q}\left(\int_{0}^{1} \lambda \omega(s) f(s, u(s)) d s\right) \\
& \left.+\mu \int_{0}^{1} g(t) \sum_{t_{k}<t} I_{k}\left(t_{k}, u\left(t_{k}\right)\right) d t\right\} .
\end{aligned}
$$

Then, the proof of sufficient is complete. 
Conversely, if $u \in E$ is a solution of (21), then we have the following.

Direct differentiation of (21) implies

$$
u^{\prime}(t)=\phi_{q}\left(\int_{t}^{1} \lambda \omega(s) f(s, u(s)) d s\right), \quad t \in J
$$

Evidently,

$$
\begin{gathered}
\left(\phi_{p}\left(u^{\prime}(t)\right)\right)^{\prime}=-\lambda \omega(t) f(t, u(t)), \\
a u(0)-b u^{\prime}(0)=\int_{0}^{1} g(t) u(t) d t, \quad u^{\prime}(1)=0 .
\end{gathered}
$$

Finally, we show that (22) holds. It is clear that $u^{\prime}(t)=$ $\phi_{q}\left(\int_{t}^{1} \lambda \omega(s) f(s, u(s)) d s\right)>0$, which implies that

$$
\|u\|=u(1), \quad \min _{t \in J} u(t)=u(0) .
$$

As we assume that $f(t, u) \geq 0$ and $\omega(t) \geq 0$, we see that any nontrivial solution $u$ of problem (7) is concave on $J$; that is, $u^{\prime \prime} \leq 0$, and then we get that $u^{\prime}(t)$ is nonincreasing on $J$.

So, for every $t \in(0,1]$, we have

$$
\frac{u(1)-u(0)}{1} \leq \frac{u(t)-u(0)}{t}
$$

that is, $u(t)-u(0) \geq t u(1)-t u(0)$. Therefore,

$$
\begin{aligned}
& \int_{0}^{1} g(t) u(t) d t-\int_{0}^{1} g(t) d t u(0) \\
& \quad \geq \int_{0}^{1} \operatorname{tg}(t) d t u(1)-\int_{0}^{1} \operatorname{tg}(t) d t u(0) .
\end{aligned}
$$

Noticing that $u$ is a positive solution of problem (7) and $a u(0)-b u^{\prime}(0)=\int_{0}^{1} g(t) u(t) d t$, we have $\int_{0}^{1} g(t) u(t) d t \leq a u(0)$. Thus, we obtain

$$
u(0) \geq \frac{\int_{0}^{1} \operatorname{tg}(t) d t}{a-\int_{0}^{1} g(t) d t+\int_{0}^{1} \operatorname{tg}(t) d t} u(1)
$$

The lemma is proved.

Define a cone $K$ in $E$ by

$$
K=\left\{u \in E: u \geq 0, \min _{t \in J} u(t) \geq \delta\|u\|\right\}
$$

where $\delta$ is defined in (23). It is easy to see that $K$ is a closed convex cone of $E$.

$$
\begin{aligned}
& \text { Define } T_{\lambda}^{\mu}: K \rightarrow E \text { by } \\
& \left(T_{\lambda}^{\mu} u\right)(t) \\
& =\int_{0}^{t} \varphi_{q}\left(\lambda \int_{s}^{1} \omega(r) f(r, u(r)) d r\right) d s \\
& +\mu \sum_{t<t_{k}} I_{k}\left(t_{k}, u\left(t_{k}\right)\right)+\frac{1}{a-\sigma} \\
& \times\left\{\int_{0}^{1} g(t)\left[\int_{0}^{t} \varphi_{q}\left(\lambda \int_{s}^{1} \omega(r) f(r, u(r)) d r\right) d s\right] d t\right. \\
& +b \varphi_{q}\left(\lambda \int_{0}^{1} \omega(s) f(s, u(s)) d s\right) \\
& \left.+\mu \int_{0}^{1} g(t) \sum_{t<t_{k}} I_{k}\left(t_{k}, u\left(t_{k}\right)\right) d t\right\} \text {. }
\end{aligned}
$$

From (40) and Lemma 10, it is easy to obtain the following result.

Lemma 11. Assume that $\left(H_{1}\right)-\left(H_{4}\right)$ hold. Problem (7) is equivalent to the fixed point problem of $T_{\lambda}^{\mu}$ in $\mathrm{K}$.

Lemma 12 (see [47, Lemmas 2.1 and 2.2]). Assume that $\left(H_{1}\right)_{-}$ $\left(H_{4}\right)$ hold. Then, $T_{\lambda}^{\mu}: K \rightarrow K$ is completely continuous.

The following well-known result of the fixed point is crucial in our arguments.

Lemma 13 (see [49]). Let $P$ be a cone in a real Banach space E. Assume that $\Omega_{1}, \Omega_{2}$ are bounded open sets in $E$ with $0 \in$ $\Omega_{1}, \bar{\Omega}_{1} \subset \Omega_{2}$. If

$$
A: P \cap\left(\bar{\Omega}_{2} \backslash \Omega_{1}\right) \rightarrow P
$$

is completely continuous such that either

(a) $\|A x\| \leq\|x\|, \forall x \in P \cap \partial \Omega_{1}$, and $\|A x\| \leq\|x\|, \forall x \in$ $P \cap \partial \Omega_{2}$, or

(b) $\|A x\| \leq\|x\|, \forall x \in P \cap \partial \Omega_{1}$, and $\|A x\| \leq\|x\|, \forall x \in$ $P \cap \partial \Omega_{2}$,

then $A$ has at least one fixed point in $P \cap\left(\bar{\Omega}_{2} \backslash \Omega_{1}\right)$.

\section{Proofs of the Main Results}

For convenience, we introduce the following notation:

$$
\gamma=\varphi_{q}\left(\int_{0}^{1} \omega(s) d s\right), \quad \sigma_{1}=\int_{t_{1}}^{1} g(s) d s .
$$

Proof of Theorem 1. Part (i). Noticing that $f(t, u)>0$, $I_{k}(t, u)>0(k=1,2, \ldots, n)$ for all $t$ and $u>0$, we can define

$$
\begin{gathered}
m_{r}=\min _{t \in J, \delta r \leq u \leq r}\{f(t, u)\}>0, \\
m^{*}=\min \left\{m_{k}, k=1,2, \ldots, n\right\}>0,
\end{gathered}
$$

where $r>0, m_{k}=\min _{t \in J, \delta r \leq u \leq r}\left\{I_{k}(t, u)\right\}, k=1,2, \ldots, n$. 
Let

$$
\lambda_{0} \geq\left(\frac{a-\sigma}{2 b \delta \gamma} r\right)^{p-1} m_{r}^{-1}, \quad \mu_{0} \geq \frac{(a-\sigma) r}{2 \sigma_{1} m^{*}} .
$$

Then, for $u \in K \cap \partial \Omega_{r}$ and $\lambda>\lambda_{0}, \mu>\mu_{0}$, we have $\left(T_{\lambda}^{\mu} u\right)(t)$

$$
\begin{aligned}
& =\int_{0}^{t} \varphi_{q}\left(\lambda \int_{s}^{1} \omega(r) f(r, u(r)) d r\right) d s \\
& +\mu \sum_{t_{k}<t} I_{k}\left(t_{k}, u\left(t_{k}\right)\right) \\
& +\frac{1}{a-\sigma}\left\{\int_{0}^{1} g(t)\right. \\
& \times\left[\int_{0}^{t} \varphi_{q}\left(\lambda \int_{s}^{1} \omega(r) f(r, u(r)) d r\right) d s\right] d t \\
& +b \varphi_{q}\left(\lambda \int_{0}^{1} \omega(s) f(s, u(s)) d s\right) \\
& \left.+\mu \int_{0}^{1} g(t) \sum_{t_{k}<t} I_{k}\left(t_{k}, u\left(t_{k}\right)\right) d t\right\} \\
& \geq \frac{b}{a-\sigma} \varphi_{q}\left(\lambda \int_{0}^{1} \omega(s) f(s, u(s)) d s\right) \\
& +\frac{1}{a-\sigma} \mu \int_{0}^{1} g(t) \sum_{t_{k}<t} I_{k}\left(t_{k}, u\left(t_{k}\right)\right) d t \\
& \geq \frac{b}{a-\sigma} \lambda^{q-1} \varphi_{q}\left(\int_{0}^{1} \omega(s) m_{r} d s\right) \\
& +\frac{\mu}{a-\sigma}\left[\int_{t_{1}}^{t_{2}} g(t) I_{1}\left(t_{1}, u\left(t_{1}\right)\right) d t\right. \\
& +\int_{t_{2}}^{t_{3}} g(t)\left(I_{1}\left(t_{1}, u\left(t_{1}\right)\right)\right. \\
& \left.+I_{2}\left(t_{2}, u\left(t_{2}\right)\right)\right) d t+\cdots \\
& +\int_{t_{n}}^{1} g(t)\left(I_{1}\left(t_{1}, u\left(t_{1}\right)\right)+I_{2}\left(t_{2}, u\left(t_{2}\right)\right)\right. \\
& \left.\left.+\cdots+I_{n}\left(t_{n}, u\left(t_{n}\right)\right)\right) d t\right] \\
& =\frac{b}{a-\sigma} \lambda^{q-1} \varphi_{q}\left(\int_{0}^{1} \omega(s) m_{r} d s\right) \\
& +\frac{\mu}{a-\sigma}\left[\int _ { t _ { 1 } } ^ { 1 } g ( t ) \left(I_{1}\left(t_{1}, u\left(t_{1}\right)\right) d t\right.\right. \\
& +\int_{t_{2}}^{1} g(t) I_{2}\left(t_{2}, u\left(t_{2}\right)\right) d t \\
& \left.\left.+\cdots+\int_{t_{n}}^{1} g(t) I_{n}\left(t_{n}, u\left(t_{n}\right)\right)\right) d t\right]
\end{aligned}
$$

$$
\begin{aligned}
\geq & \frac{b}{a-\sigma} \lambda^{q-1} \varphi_{q}\left(\int_{0}^{1} \omega(s) m_{r} d s\right) \\
& +\frac{\mu}{a-\sigma} \int_{t_{1}}^{1} g(t) I_{1}\left(t_{1}, u\left(t_{1}\right)\right) d t \\
\geq & \frac{b}{a-\sigma} \lambda^{q-1} m_{r}^{q-1} \gamma+\frac{\sigma_{1}}{a-\sigma} \mu m^{*} \\
> & \frac{b}{a-\sigma} \lambda_{0}^{1-q} m_{r}^{q-1} \gamma+\frac{\sigma_{1}}{a-\sigma} \mu_{0} m^{*} \\
\geq & \frac{1}{2} r+\frac{1}{2} r=r,
\end{aligned}
$$

which implies that

$$
\begin{gathered}
\left\|T_{\lambda}^{\mu} u\right\|>\|u\|, \quad \forall u \in K \cap \partial \Omega_{r}, \\
\lambda>\lambda_{0}, \quad \mu>\mu_{0} .
\end{gathered}
$$

If $0<f^{\infty}<+\infty, 0<I^{\infty}<+\infty$, then there exist $l_{1}>0$, and $l_{2}>0$ and $R>r>0$ such that

$$
\begin{array}{r}
f(t, u)<l_{1} \varphi_{p}(u), \quad I_{k}(t, u)<l_{2} u, \\
(\forall t \in J, \quad u \geq R, \quad k=1,2, \ldots, n),
\end{array}
$$

where $l_{1}$ satisfies

$$
\frac{2(a+b)}{a-\sigma} \varphi_{q}(\lambda) \varphi_{q}\left(l_{1}\right) \gamma \leq 1
$$

$l_{2}$ satisfies

$$
\frac{2 a}{a-\sigma} \mu n l_{2} \leq 1
$$

Let $v=R / \delta$. Thus, when $u \in K \cap \partial \Omega_{v}$, we have

$$
u(t) \geq \delta\|u\|=\delta v=R, \quad t \in J
$$

and then we get

$$
\begin{aligned}
& \left(T_{\lambda}^{\mu} u\right)(t) \\
& =\int_{0}^{t} \varphi_{q}\left(\lambda \int_{s}^{1} \omega(r) f(r, u(r)) d r\right) d s+\mu \sum_{t<t_{k}} I_{k}\left(t_{k}, u\left(t_{k}\right)\right) \\
& +\frac{1}{a-\sigma}\left\{\int_{0}^{1} g(t)\right. \\
& \times\left[\int_{0}^{t} \varphi_{q}\left(\lambda \int_{s}^{1} \omega(r) f(r, u(r)) d r\right) d s\right] d t \\
& +b \varphi_{q}\left(\lambda \int_{0}^{1} \omega(s) f(s, u(s)) d s\right) \\
& \left.+\mu \int_{0}^{1} g(t) \sum_{t<t_{k}} I_{k}\left(t_{k}, u\left(t_{k}\right)\right) d t\right\}
\end{aligned}
$$




$$
\begin{aligned}
& \leq \int_{0}^{1} \varphi_{q}\left(\lambda \int_{s}^{1} \omega(r) f(r, u(r)) d r\right) d s \\
& +\frac{1}{a-\sigma}\left\{\int_{0}^{1} g(t)\right. \\
& \times\left[\int_{0}^{1} \varphi_{q}\left(\lambda \int_{s}^{1} \omega(r) f(r, u(r)) d r\right) d s\right] d t \\
& \left.+b \varphi_{q}\left(\lambda \int_{0}^{1} \omega(s) f(s, u(s)) d s\right)\right\} \\
& +\mu \sum_{k=1}^{n} I_{k}\left(t_{k}, u\left(t_{k}\right)\right) \\
& +\frac{1}{a-\sigma} \mu \int_{0}^{1} g(t) \sum_{k=1}^{n} I_{k}\left(t_{k}, u\left(t_{k}\right)\right) d t \\
& =\int_{0}^{1} \varphi_{q}\left(\lambda \int_{s}^{1} \omega(r) f(r, u(r)) d r\right) d s\left(1+\frac{\sigma}{a-\sigma}\right) \\
& +\frac{b}{a-\sigma} \varphi_{q}\left(\lambda \int_{0}^{1} \omega(s) f(s, u(s)) d s\right) \\
& +\mu \sum_{k=1}^{n} I_{k}\left(t_{k}, u\left(t_{k}\right)\right) \\
& +\frac{1}{a-\sigma} \mu \int_{0}^{1} g(t) \sum_{k=1}^{n} I_{k}\left(t_{k}, u\left(t_{k}\right)\right) d t \\
& \leq \frac{a}{a-\sigma} \int_{0}^{1} \varphi_{q}\left(\lambda \int_{0}^{1} \omega(r) f(r, u(r)) d r\right) d s \\
& +\frac{b}{a-\sigma} \varphi_{q}\left(\lambda \int_{0}^{1} \omega(s) f(s, u(s)) d s\right) \\
& +\mu \sum_{k=1}^{n} I_{k}\left(t_{k}, u\left(t_{k}\right)\right) \\
& +\frac{1}{a-\sigma} \mu \int_{0}^{1} g(t) \sum_{k=1}^{n} I_{k}\left(t_{k}, u\left(t_{k}\right)\right) d t \\
& =\frac{a+b}{a-\sigma} \varphi_{q}\left(\lambda \int_{0}^{1} \omega(s) f(s, u(s)) d s\right) \\
& +\mu \sum_{k=1}^{n} I_{k}\left(t_{k}, u\left(t_{k}\right)\right) \\
& +\frac{1}{a-\sigma} \mu \int_{0}^{1} g(t) \sum_{k=1}^{n} I_{k}\left(t_{k}, u\left(t_{k}\right)\right) d t \\
& \leq \frac{a+b}{a-\sigma} \varphi_{q}\left(\lambda \int_{0}^{1} \omega(s) l_{1} \varphi_{p}(u(s)) d s\right) \\
& +\mu \sum_{k=1}^{n} l_{2} u\left(t_{k}\right) \\
& +\frac{1}{a-\sigma} \mu \int_{0}^{1} g(t) \sum_{k=1}^{n} l_{2} u\left(t_{k}\right) d t
\end{aligned}
$$$$
\leq \frac{a+b}{a-\sigma} \varphi_{q}\left(l_{1}\right)\|u\| \varphi_{q}\left(\lambda \int_{0}^{1} \omega(s) d s\right)
$$

This yields

$$
\left\|T_{\lambda}^{\mu} u\right\| \leq\|u\|, \quad \forall u \in K \cap \partial \Omega_{v}
$$

Applying (b) of Lemma 13 to (46) and (52) yields that $T$ has a fixed point $u \in K \cap\left(\bar{\Omega}_{v} \backslash \Omega_{r}\right)$ with $r \leq\|u\| \leq v=(1 / \delta) R$. Hence, since for $u \in K$ we have $u(t) \geq \delta\|u\|$ for $t \in J$, it follows that (12) holds. This gives the proof of Part (i).

Part (ii). Noticing that $f(t, u)>0, I_{k}(t, u)>0$ for all $t$ and $u>0$, we can define

$$
\begin{gathered}
M_{r}=\max _{t \in J, 0 \leq u \leq r}\{f(t, u)\}>0, \\
M^{*}=\max \left\{M_{k}, k=1,2, \ldots, n\right\}>0,
\end{gathered}
$$

where $r>0, M_{k}=\max _{t \in J, 0 \leq u \leq r}\left\{I_{k}(t, u)\right\}, k=1,2, \ldots, n$.

Let

$$
\bar{\lambda}_{0} \leq\left(\frac{(a-\sigma) r}{2(a+b) \gamma}\right)^{p-1} M_{r}^{-1}, \quad \bar{\mu}_{0} \leq \frac{(a-\sigma) r}{2 a n M^{*}}
$$

Then, for $u \in K \cap \partial \Omega_{r}$ and $\lambda<\bar{\lambda}_{0}, \mu<\bar{\mu}_{0}$, we have

$$
\begin{gathered}
\left(T_{\lambda}^{\mu} u\right)(t) \\
=\int_{0}^{t} \varphi_{q}\left(\lambda \int_{s}^{1} \omega(r) f(r, u(r)) d r\right) d s \\
+\mu \sum_{t<t_{k}} I_{k}\left(t_{k}, u\left(t_{k}\right)\right) \\
+\frac{1}{a-\sigma}\left\{\int_{0}^{1} g(t)\right. \\
\times\left[\int_{0}^{t} \varphi_{q}\left(\lambda \int_{s}^{1} \omega(r) f(r, u(r)) d r\right) d s\right] d t \\
+b \varphi_{q}\left(\lambda \int_{0}^{1} \omega(s) f(s, u(s)) d s\right) \\
\left.+\mu \int_{0}^{1} g(t) \sum_{t<t_{k}} I_{k}\left(t_{k}, u\left(t_{k}\right)\right) d t\right\}
\end{gathered}
$$




$$
\begin{aligned}
& \leq \int_{0}^{1} \varphi_{q}\left(\lambda \int_{s}^{1} \omega(r) f(r, u(r)) d r\right) d s \\
& +\frac{1}{a-\sigma}\left\{\int_{0}^{1} g(t)\right. \\
& \times\left[\int_{0}^{1} \varphi_{q}\left(\lambda \int_{s}^{1} \omega(r) f(r, u(r)) d r\right) d s\right] d t \\
& \left.+b \varphi_{q}\left(\lambda \int_{0}^{1} \omega(s) f(s, u(s)) d s\right)\right\} \\
& +\mu \sum_{k=1}^{n} I_{k}\left(t_{k}, u\left(t_{k}\right)\right) \\
& +\frac{1}{a-\sigma} \mu \int_{0}^{1} g(t) \sum_{k=1}^{n} I_{k}\left(t_{k}, u\left(t_{k}\right)\right) d t \\
& =\int_{0}^{1} \varphi_{q}\left(\lambda \int_{s}^{1} \omega(r) f(r, u(r)) d r\right) d s\left(1+\frac{\sigma}{a-\sigma}\right) \\
& +\frac{b}{a-\sigma} \varphi_{q}\left(\lambda \int_{0}^{1} \omega(s) f(s, u(s)) d s\right) \\
& +\mu \sum_{k=1}^{n} I_{k}\left(t_{k}, u\left(t_{k}\right)\right) \\
& +\frac{1}{a-\sigma} \mu \int_{0}^{1} g(t) \sum_{k=1}^{n} I_{k}\left(t_{k}, u\left(t_{k}\right)\right) d t \\
& \leq \frac{a}{a-\sigma} \int_{0}^{1} \varphi_{q}\left(\lambda \int_{0}^{1} \omega(r) f(r, u(r)) d r\right) d s \\
& +\frac{b}{a-\sigma} \varphi_{q}\left(\lambda \int_{0}^{1} \omega(s) f(s, u(s)) d s\right) \\
& +\mu \sum_{k=1}^{n} I_{k}\left(t_{k}, u\left(t_{k}\right)\right) \\
& +\frac{1}{a-\sigma} \mu \int_{0}^{1} g(t) \sum_{k=1}^{n} I_{k}\left(t_{k}, u\left(t_{k}\right)\right) d t \\
& =\frac{a+b}{a-\sigma} \varphi_{q}\left(\lambda \int_{0}^{1} \omega(s) f(s, u(s)) d s\right) \\
& +\mu \sum_{k=1}^{n} I_{k}\left(t_{k}, u\left(t_{k}\right)\right) \\
& +\frac{1}{a-\sigma} \mu \int_{0}^{1} g(t) \sum_{k=1}^{n} I_{k}\left(t_{k}, u\left(t_{k}\right)\right) d t \\
& \leq \frac{a+b}{a-\sigma} \varphi_{q}\left(\lambda \int_{0}^{1} \omega(s) M_{r} d s\right) \\
& +\mu \sum_{k=1}^{n} M^{*}+\frac{1}{a-\sigma} \mu \int_{0}^{1} g(t) \sum_{k=1}^{n} M^{*} d t \\
& \leq \frac{a+b}{a-\sigma} \varphi_{q}(\lambda) \varphi_{q}\left(M_{r}\right) \gamma+\frac{a}{a-\sigma} \mu n M^{*}
\end{aligned}
$$$$
<\frac{a+b}{a-\sigma} \varphi_{q}\left(\bar{\lambda}_{0}\right) \varphi_{q}\left(M_{r}\right) \gamma+\frac{a}{a-\sigma} \bar{\mu}_{0} n M^{*}
$$$$
\leq \frac{1}{2}\|u\|+\frac{1}{2}\|u\|=\|u\| \text {. }
$$

This implies that

$$
\left\|T_{\lambda}^{\mu} u\right\|<\|u\|, \quad \forall u \in K \cap \partial \Omega_{r}
$$

If $0<f_{\infty}<+\infty, 0<I_{\infty}<+\infty$, then there exist $l_{3}>$ 0 , and $l_{4}>0$ and $R>r>0$ such that

$$
\begin{array}{r}
f(t, u)>l_{3} \varphi_{p}(u), \quad I_{k}(t, u)>l_{4} u, \\
(\forall t \in J, u \geq R, k=1,2, \ldots, n),
\end{array}
$$

where $l_{3}$ satisfies

$$
\frac{2 b}{a-\sigma} \lambda^{q-1} l_{3}^{q-1} \gamma \delta \geq 1
$$

$l_{4}$ satisfies

$$
\frac{2 \sigma_{1}}{a-\sigma} \mu l_{4} \delta \geq 1
$$

Let $\nu=R / \delta$. Then, for $u \in K \cap \partial \Omega_{\nu}$, we obtain

$$
u(t) \geq \delta R=v, \quad t \in J,
$$

and it follows from (40) that

$$
\begin{aligned}
& \left(T_{\lambda}^{\mu} u\right)(t) \\
& =\int_{0}^{t} \varphi_{q}\left(\lambda \int_{s}^{1} \omega(r) f\left(r, u_{1}(r)\right) d r\right) d s \\
& +\mu \sum_{t<t_{k}} I_{k}\left(t_{k}, u\left(t_{k}\right)\right) \\
& +\frac{1}{a-\sigma}\left\{\int_{0}^{1} g(t)\right. \\
& \times\left[\int_{0}^{t} \varphi_{q}\left(\lambda \int_{s}^{1} \omega(r) f\left(r, u_{1}(r)\right) d r\right) d s\right] d t \\
& +b \varphi_{q}\left(\lambda \int_{0}^{1} \omega(s) f\left(s, u_{1}(s)\right) d s\right) \\
& \left.+\mu \int_{0}^{1} g(t) \sum_{t<t_{k}} I_{k}\left(t_{k}, u\left(t_{k}\right)\right) d t\right\} \\
& \geq \frac{b}{a-\sigma} \varphi_{q}\left(\lambda \int_{0}^{1} \omega(s) f\left(s, u_{1}(s)\right) d s\right) \\
& +\frac{1}{a-\sigma} \mu \int_{0}^{1} g(t) \sum_{t<t_{k}} I_{k}\left(t_{k}, u\left(t_{k}\right)\right) d t
\end{aligned}
$$




$$
\begin{aligned}
\geq & \frac{b}{a-\sigma} \varphi_{q}\left(\lambda \int_{0}^{1} \omega(s) f\left(s, u_{1}(s)\right) d s\right) \\
& +\frac{1}{a-\sigma} \mu \int_{t_{1}}^{1} g(t) I_{1}\left(t_{1}, u\left(t_{1}\right)\right) d t \\
\geq & \frac{b}{a-\sigma} \lambda^{q-1} \varphi_{q}\left(\int_{0}^{1} \omega(s) l_{3} \varphi_{p}(u(s)) d s\right) \\
& +\frac{\mu}{a-\sigma} \int_{t_{1}}^{1} g(t) l_{4} u\left(t_{1}\right) d t \\
\geq & \frac{b}{a-\sigma} \lambda^{q-1} l_{3}^{q-1} \gamma \delta\|u\| \\
& +\frac{\sigma_{1}}{a-\sigma} \mu l_{4} \delta\|u\| \\
\geq & \frac{1}{2}\|u\|+\frac{1}{2}\|u\|=\|u\|,
\end{aligned}
$$

which implies that

$$
\left\|T_{\lambda}^{\mu} u\right\|>\|u\|, \quad \forall u \in K \cap \partial \Omega_{\nu} .
$$

Applying (a) of Lemma 13 to (56) and (62) yields that $T_{\lambda}^{\mu}$ has a fixed point $u \in K \cap\left(\bar{\Omega}_{\nu} \backslash \Omega_{r}\right)$ with $r \leq\|u\| \leq v=(1 / \delta) R$. Hence, since for $u \in K$ we have $u(t) \geq \delta\|u\|$ for $t \in J$, it follows that (13) holds. This finishes the proof of Part (ii).

Proof of Theorem 2. Part (i). Noticing that $f(t, u)>0$, $I_{k}(t, u)>0(k=1,2, \ldots, n)$ for all $t$ and $u>0$, we can define

$$
\begin{gathered}
m_{R}=\min _{t \in J, \delta R \leq u \leq R}\{f(t, u)\}>0, \\
m_{R}^{*}=\min \left\{m_{R k}, k=1,2, \ldots, n\right\}>0,
\end{gathered}
$$

where $R>0, m_{R k}=\min _{t \in J, \delta R \leq u \leq R}\left\{I_{k}(t, u)\right\}, k=1,2, \ldots, n$.

Let

$$
\lambda_{0} \geq\left(\frac{a-\sigma}{2 b \delta \gamma} R\right)^{p-1} m_{R}^{-1}, \quad \mu_{0} \geq \frac{(a-\sigma) R}{2 \sigma_{1} m_{R}^{*}} .
$$

Then, for $u \in K \cap \partial \Omega_{R}$ and $\lambda>\lambda_{0}, \mu>\mu_{0}$, we have

$$
\begin{aligned}
& \left(T_{\lambda}^{\mu} u\right)(t) \\
& =\int_{0}^{t} \varphi_{q}\left(\lambda \int_{s}^{1} \omega(r) f(r, u(r)) d r\right) d s \\
& +\mu \sum_{t<t_{k}} I_{k}\left(t_{k}, u\left(t_{k}\right)\right) \\
& +\frac{1}{a-\sigma}\left\{\int_{0}^{1} g(t)\right. \\
& \times\left[\int_{0}^{t} \varphi_{q}\left(\lambda \int_{s}^{1} \omega(r) f(r, u(r)) d r\right) d s\right] d t \\
& +b \varphi_{q}\left(\lambda \int_{0}^{1} \omega(s) f(s, u(s)) d s\right) \\
& \left.+\mu \int_{0}^{1} g(t) \sum_{t<t_{k}} I_{k}\left(t_{k}, u\left(t_{k}\right)\right) d t\right\}
\end{aligned}
$$

$$
\begin{aligned}
\geq & \frac{b}{a-\sigma} \varphi_{q}\left(\lambda \int_{0}^{1} \omega(s) f(s, u(s)) d s\right) \\
& +\frac{1}{a-\sigma} \mu \int_{0}^{1} g(t) \sum_{t<t_{k}} I_{k}\left(t_{k}, u\left(t_{k}\right)\right) d t \\
\geq & \frac{b}{a-\sigma} \varphi_{q}\left(\lambda \int_{0}^{1} \omega(s) f(s, u(s)) d s\right) \\
& +\frac{1}{a-\sigma} \mu \int_{t_{1}}^{1} g(t) I_{1}\left(t_{1}, u\left(t_{1}\right)\right) d t \\
\geq & \frac{b}{a-\sigma} \lambda^{q-1} \varphi_{q}\left(\int_{0}^{1} \omega(s) m_{R} d s\right) \\
& +\frac{\mu}{a-\sigma} \int_{t_{1}}^{1} g(t) m_{R}^{*} d t \\
\geq & \frac{b}{a-\sigma} \lambda^{q-1} m_{R}^{q-1} \gamma+\frac{\sigma_{1}}{a-\sigma} \mu m_{R}^{*} \\
> & \frac{b}{a-\sigma} \lambda_{0}^{1-q} m_{R}^{q-1} \gamma+\frac{\sigma_{1}}{a-\sigma} \mu_{0} m_{R}^{*} \\
\geq & \frac{1}{2} R+\frac{1}{2} R=R, \\
& \\
& \\
& \\
&
\end{aligned}
$$

which implies that

$$
\begin{gathered}
\left\|T_{\lambda}^{\mu} u\right\|>\|u\|, \quad \forall u \in K \cap \partial \Omega_{R}, \\
\lambda>\lambda_{0}, \quad \mu>\mu_{0} .
\end{gathered}
$$

If $0<f^{0}<+\infty, 0<I^{0}<+\infty$, then there exist $l_{1}>0$, $l_{2}>0$ and $0<r<R$ such that

$$
\begin{gathered}
f(t, u)<l_{1} \varphi_{p}(u), \quad I_{k}(t, u)<l_{2} u, \\
(\forall t \in J, 0 \leq u \leq r, 2 k=1,2, \ldots, n),
\end{gathered}
$$

where $l_{1}$ and $l_{2}$ satisfy (48) and (49), respectively.

Therefore, for $u \in K \cap \partial \Omega_{r}$, we have

$$
\begin{aligned}
& \left(T_{\lambda}^{\mu} u\right)(t) \\
& =\int_{0}^{t} \varphi_{q}\left(\lambda \int_{s}^{1} \omega(r) f(r, u(r)) d r\right) d s \\
& +\mu \sum_{t<t_{k}} I_{k}\left(t_{k}, u\left(t_{k}\right)\right) \\
& +\frac{1}{a-\sigma}\left\{\int_{0}^{1} g(t)\right. \\
& \times\left[\int_{0}^{t} \varphi_{q}\left(\lambda \int_{s}^{1} \omega(r) f(r, u(r)) d r\right) d s\right] d t \\
& +b \varphi_{q}\left(\lambda \int_{0}^{1} \omega(s) f(s, u(s)) d s\right) \\
& \left.+\mu \int_{0}^{1} g(t) \sum_{t<t_{k}} I_{k}\left(t_{k}, u\left(t_{k}\right)\right) d t\right\}
\end{aligned}
$$




$$
\begin{aligned}
& \leq \int_{0}^{1} \varphi_{q}\left(\lambda \int_{s}^{1} \omega(r) f(r, u(r)) d r\right) d s \\
& +\frac{1}{a-\sigma}\left\{\int_{0}^{1} g(t)\right. \\
& \times\left[\int_{0}^{1} \varphi_{q}\left(\lambda \int_{s}^{1} \omega(r) f(r, u(r)) d r\right) d s\right] d t \\
& \left.+b \varphi_{q}\left(\lambda \int_{0}^{1} \omega(s) f(s, u(s)) d s\right)\right\} \\
& +\mu \sum_{k=1}^{n} I_{k}\left(t_{k}, u\left(t_{k}\right)\right) \\
& +\frac{1}{a-\sigma} \mu \int_{0}^{1} g(t) \sum_{k=1}^{n} I_{k}\left(t_{k}, u\left(t_{k}\right)\right) d t \\
& =\int_{0}^{1} \varphi_{q}\left(\lambda \int_{s}^{1} \omega(r) f(r, u(r)) d r\right) d s\left(1+\frac{\sigma}{a-\sigma}\right) \\
& +\frac{b}{a-\sigma} \varphi_{q}\left(\lambda \int_{0}^{1} \omega(s) f(s, u(s)) d s\right) \\
& +\mu \sum_{k=1}^{n} I_{k}\left(t_{k}, u\left(t_{k}\right)\right) \\
& +\frac{1}{a-\sigma} \mu \int_{0}^{1} g(t) \sum_{k=1}^{n} I_{k}\left(t_{k}, u\left(t_{k}\right)\right) d t \\
& \leq \frac{a}{a-\sigma} \int_{0}^{1} \varphi_{q}\left(\lambda \int_{0}^{1} \omega(r) f(r, u(r)) d r\right) d s \\
& +\frac{b}{a-\sigma} \varphi_{q}\left(\lambda \int_{0}^{1} \omega(s) f(s, u(s)) d s\right) \\
& +\mu \sum_{k=1}^{n} I_{k}\left(t_{k}, u\left(t_{k}\right)\right) \\
& +\frac{1}{a-\sigma} \mu \int_{0}^{1} g(t) \sum_{k=1}^{n} I_{k}\left(t_{k}, u\left(t_{k}\right)\right) d t \\
& =\frac{a+b}{a-\sigma} \varphi_{q}\left(\lambda \int_{0}^{1} \omega(s) f(s, u(s)) d s\right) \\
& +\mu \sum_{k=1}^{n} I_{k}\left(t_{k}, u\left(t_{k}\right)\right) \\
& +\frac{1}{a-\sigma} \mu \int_{0}^{1} g(t) \sum_{k=1}^{n} I_{k}\left(t_{k}, u\left(t_{k}\right)\right) d t \\
& \leq \frac{a+b}{a-\sigma} \varphi_{q}\left(\lambda \int_{0}^{1} \omega(s) l_{1} \varphi_{p}(u(s)) d s\right) \\
& +\mu \sum_{k=1}^{n} l_{2} u\left(t_{k}\right) \\
& +\frac{1}{a-\sigma} \mu \int_{0}^{1} g(t) \sum_{k=1}^{n} l_{2} u\left(t_{k}\right) d t
\end{aligned}
$$$$
\leq \frac{a+b}{a-\sigma} \varphi_{q}\left(l_{1}\right)\|u\| \varphi_{q}\left(\lambda \int_{0}^{1} \omega(s) d s\right)
$$

This yields

$$
\left\|T_{\lambda}^{\mu} u\right\| \leq\|u\|, \quad \forall u \in K \cap \partial \Omega_{r}
$$

Applying (a) of Lemma 13 to (66) and (69) yields that $T_{\lambda}^{\mu}$ has a fixed point $u \in K \cap\left(\bar{\Omega}_{R} \backslash \Omega_{r}\right)$ with $r \leq\|u\| \leq R$. Hence, since for $u \in K$ we have $u(t) \geq \delta\|u\|$ for $t \in J$, it follows that (13) holds. This gives the proof of Part (i).

Part (ii). Noticing that $f(t, u)>0, I_{k}(t, u)>0(k=$ $1,2, \ldots, n)$ for all $t$ and $u>0$, we can define

$$
\begin{gathered}
M_{R}=\max _{t \in J, 0 \leq u \leq R}\{f(t, u)\}>0, \\
M_{R}^{*}=\max \left\{M_{R k}, k=1,2, \ldots, n\right\}>0,
\end{gathered}
$$

where $R>0, M_{R k}=\max _{t \in J, 0 \leq u \leq R}\left\{I_{k}(t, u)\right\}, k=1,2, \ldots, n$.

Let

$$
\bar{\lambda}_{0} \leq\left(\frac{a-\sigma}{2(a+b) \gamma} R\right)^{p-1} M_{R}^{-1}, \quad \bar{\mu}_{0} \leq \frac{(a-\sigma) R}{2 \sigma n M_{R}^{*}} .
$$

Then, for $u \in K \cap \partial \Omega_{R}$ and $\lambda<\bar{\lambda}_{0}, \mu<\bar{\mu}_{0}$, we have

$$
\begin{aligned}
& \left(T_{\lambda}^{\mu} u\right)(t) \\
& =\int_{0}^{t} \varphi_{q}\left(\lambda \int_{s}^{1} \omega(r) f(r, u(r)) d r\right) d s \\
& +\mu \sum_{t<t_{k}} I_{k}\left(t_{k}, u\left(t_{k}\right)\right) \\
& +\frac{1}{a-\sigma}\left\{\int_{0}^{1} g(t)\right. \\
& \quad \times\left[\int_{0}^{t} \varphi_{q}\left(\lambda \int_{s}^{1} \omega(r) f(r, u(r)) d r\right) d s\right] d t \\
& +b \varphi_{q}\left(\lambda \int_{0}^{1} \omega(s) f(s, u(s)) d s\right) \\
& \left.+\mu \int_{0}^{1} g(t) \sum_{t<t_{k}} I_{k}\left(t_{k}, u\left(t_{k}\right)\right) d t\right\}
\end{aligned}
$$




$$
\begin{aligned}
& \leq \int_{0}^{1} \varphi_{q}\left(\lambda \int_{s}^{1} \omega(r) f(r, u(r)) d r\right) d s \\
& +\frac{1}{a-\sigma}\left\{\int_{0}^{1} g(t)\right. \\
& \times\left[\int_{0}^{1} \varphi_{q}\left(\lambda \int_{s}^{1} \omega(r) f(r, u(r)) d r\right) d s\right] d t \\
& \left.+b \varphi_{q}\left(\lambda \int_{0}^{1} \omega(s) f(s, u(s)) d s\right)\right\} \\
& +\mu \sum_{k=1}^{n} I_{k}\left(t_{k}, u\left(t_{k}\right)\right) \\
& +\frac{1}{a-\sigma} \mu \int_{0}^{1} g(t) \sum_{k=1}^{n} I_{k}\left(t_{k}, u\left(t_{k}\right)\right) d t \\
& =\int_{0}^{1} \varphi_{q}\left(\lambda \int_{s}^{1} \omega(r) f(r, u(r)) d r\right) d s\left(1+\frac{\sigma}{a-\sigma}\right) \\
& +\frac{b}{a-\sigma} \varphi_{q}\left(\lambda \int_{0}^{1} \omega(s) f(s, u(s)) d s\right) \\
& +\mu \sum_{k=1}^{n} I_{k}\left(t_{k}, u\left(t_{k}\right)\right) \\
& +\frac{1}{a-\sigma} \mu \int_{0}^{1} g(t) \sum_{k=1}^{n} I_{k}\left(t_{k}, u\left(t_{k}\right)\right) d t \\
& \leq \frac{a}{a-\sigma} \int_{0}^{1} \varphi_{q}\left(\lambda \int_{0}^{1} \omega(r) f(r, u(r)) d r\right) d s \\
& +\frac{b}{a-\sigma} \varphi_{q}\left(\lambda \int_{0}^{1} \omega(s) f(s, u(s)) d s\right) \\
& +\mu \sum_{k=1}^{n} I_{k}\left(t_{k}, u\left(t_{k}\right)\right) \\
& +\frac{1}{a-\sigma} \mu \int_{0}^{1} g(t) \sum_{k=1}^{n} I_{k}\left(t_{k}, u\left(t_{k}\right)\right) d t \\
& =\frac{a+b}{a-\sigma} \varphi_{q}\left(\lambda \int_{0}^{1} \omega(s) f(s, u(s)) d s\right) \\
& +\mu \sum_{k=1}^{n} I_{k}\left(t_{k}, u\left(t_{k}\right)\right) \\
& +\frac{1}{a-\sigma} \mu \int_{0}^{1} g(t) \sum_{k=1}^{n} I_{k}\left(t_{k}, u\left(t_{k}\right)\right) d t \\
& \leq \frac{a+b}{a-\sigma} \varphi_{q}\left(\lambda \int_{0}^{1} \omega(s) M_{R} d s\right) \\
& +\mu \sum_{k=1}^{n} M_{R}^{*}+\frac{1}{a-\sigma} \mu \int_{0}^{1} g(t) \sum_{k=1}^{n} M_{R}^{*} d t \\
& \leq \frac{a+b}{a-\sigma} \varphi_{q}(\lambda) \varphi_{q}\left(M_{R}\right) \gamma+\frac{a}{a-\sigma} \mu n M_{R}^{*}
\end{aligned}
$$$$
<\frac{a+b}{a-\sigma} \varphi_{q}\left(\bar{\lambda}_{0}\right) \varphi_{q}\left(M_{R}\right) \gamma+\frac{a}{a-\sigma} \bar{\mu}_{0} n M_{R}^{*}
$$$$
\leq \frac{1}{2} R+\frac{1}{2} R=R
$$

This implies that

$$
\left\|T_{\lambda}^{\mu} u\right\|<\|u\|, \quad \forall u \in K \cap \partial \Omega_{R} .
$$

If $0<f_{0}<+\infty, 0<I_{0}<+\infty$, then there exist $l_{3}>0, l_{4}>$ 0 and $0<r<R$ such that

$$
\begin{array}{r}
f(t, u)>l_{3} \varphi_{p}(u), \quad I_{k}(t, u)>l_{4} u \\
(\forall t \in J, 0 \leq u \leq r, k=1,2, \ldots, n),
\end{array}
$$

where $l_{3}$ and $l_{4}$ satisfy (58) and (59), respectively.

Therefore, for $u \in K \cap \partial \Omega_{r}$, we obtain

$$
\begin{aligned}
& \left(T_{\lambda}^{\mu} u\right)(t) \\
& =\int_{0}^{t} \varphi_{q}\left(\lambda \int_{s}^{1} \omega(r) f(r, u(r)) d r\right) d s \\
& +\mu \sum_{t<t_{k}} I_{k}\left(t_{k}, u\left(t_{k}\right)\right) \\
& +\frac{1}{a-\sigma}\left\{\int_{0}^{1} g(t)\right. \\
& \times\left[\int_{0}^{t} \varphi_{q}\left(\lambda \int_{s}^{1} \omega(r) f(r, u(r)) d r\right) d s\right] d t \\
& +b \varphi_{q}\left(\lambda \int_{0}^{1} \omega(s) f(s, u(s)) d s\right) \\
& \left.+\mu \int_{0}^{1} g(t) \sum_{t<t_{k}} I_{k}\left(t_{k}, u\left(t_{k}\right)\right) d t\right\} \\
& \geq \frac{b}{a-\sigma} \varphi_{q}\left(\lambda \int_{0}^{1} \omega(s) f(s, u(s)) d s\right) \\
& +\frac{1}{a-\sigma} \mu \int_{0}^{1} g(t) \sum_{t<t_{k}} I_{k}\left(t_{k}, u\left(t_{k}\right)\right) d t \\
& \geq \frac{b}{a-\sigma} \varphi_{q}\left(\lambda \int_{0}^{1} \omega(s) f(s, u(s)) d s\right) \\
& +\frac{1}{a-\sigma} \mu \int_{t_{1}}^{1} g(t) I_{1}\left(t_{1}, u\left(t_{1}\right)\right) d t \\
& \geq \frac{b}{a-\sigma} \lambda^{q-1} \varphi_{q}\left(\int_{0}^{1} \omega(s) l_{3} \varphi_{p}(u(s)) d s\right) \\
& +\frac{\mu}{a-\sigma} \int_{t_{1}}^{1} g(t) l_{4} u\left(t_{k}\right) d t \\
& \geq \frac{b}{a-\sigma} \lambda^{q-1} l_{3}^{q-1} \gamma \delta\|u\| \\
& +\frac{\sigma_{1}}{a-\sigma} \mu k l_{4} \delta\|u\| \\
& \geq \frac{1}{2}\|u\|+\frac{1}{2}\|u\|=\|u\|,
\end{aligned}
$$


which implies that

$$
\left\|T_{\lambda}^{\mu} u\right\|>\|u\|, \quad \forall u \in K \cap \partial \Omega_{r} .
$$

Applying (a) of Lemma 13 to (73) and (76) yields that $T_{\lambda}^{\mu}$ has a fixed point $u \in K \cap\left(\bar{\Omega}_{R} \backslash \Omega_{r}\right)$ with $r \leq\|u\| \leq R$. Hence, since for $u \in K$ we have $u(t) \geq \delta\|u\|$ for $t \in J$, it follows that (13) holds. This finishes the proof of Part (ii).

Proof of Theorem 5. Similar to the proof of Theorems 1(i) and 2(i), respectively, one can show that Theorems 5(i) and (ii) hold.

Considering Part (iii), choose two numbers $r_{1}$ and $r_{2}$ satisfying (11). By Theorems 1(i) and 2(i), there exist $\lambda_{0}>0$ and $\mu_{0}>0$ such that

$$
\left\|T_{\lambda}^{\mu} u\right\|>\|u\|, \quad \forall u \in K \cap \partial \Omega_{r_{i}}, \quad i=1,2 .
$$

Since $f^{0}=f^{\infty}=I^{\infty}=I^{0}=0$, from the proof of Theorem 1(i) and Theorem 2(i) and from (11), it follows that

$$
\begin{gathered}
\left\|T_{\lambda}^{\mu} u\right\|<\|u\|, \quad \forall u \in K \cap \partial \Omega_{r}, \\
\left\|T_{\lambda}^{\mu} u\right\|<\|u\|, \quad \forall u \in K \cap \partial \Omega_{R} .
\end{gathered}
$$

Applying Lemma 12 to (77)-(79) yields that $T_{\lambda}^{\mu}$ has two fixed points $u_{1}$ and $u_{2}$ such that $u_{1} \in K \cap\left(\bar{\Omega}_{r_{1}} \backslash \Omega_{r}\right)$ and $u_{2} \in K \cap\left(\bar{\Omega}_{R} \backslash \Omega_{r_{2}}\right)$. These are the desired distinct positive solutions of problem (7) for $\lambda_{0}>0$ and $\mu_{0}>0$ satisfying (17). Then, the result of Part (iii) follows.

Proof of Theorem 6. Similar to the proof of Theorems 1(ii) and 2(ii), respectively, one can show that Theorems 6(i) and (ii) hold.

Now, considering Part (iii), choose two numbers $r_{1}$ and $r_{2}$ satisfying (11). By Theorems 1(ii) and 2(ii), there exist $\bar{\lambda}_{0}>0$ and $\bar{\mu}_{0}>0$ such that

$$
\begin{gathered}
\left\|T_{\lambda}^{\mu} u\right\|<\|u\|, \quad \forall 0<\lambda<\bar{\lambda}_{0}, \\
0<\mu<\bar{\mu}_{0}, \quad u \in K \cap \partial \Omega_{r_{i}}, \quad i=1,2 .
\end{gathered}
$$

Since $f_{0}=f_{\infty}=I_{\infty}=I_{0}=\infty$, from the proof of Theorems 1(ii) and 2(ii) and from (11), it follows that

$$
\begin{gathered}
\left\|T_{\lambda}^{\mu} u\right\|>\|u\|, \quad \forall u \in K \cap \partial \Omega_{r}, \\
\left\|T_{\lambda}^{\mu} u\right\|>\|u\|, \quad \forall u \in K \cap \partial \Omega_{R} .
\end{gathered}
$$

Applying Lemma 13 to (80)-(82) yields that $T_{\lambda}^{\mu}$ has two fixed points $u_{1}$ and $u_{2}$ such that $u_{1} \in K \cap\left(\bar{\Omega}_{r_{1}} \backslash \Omega_{r}\right)$ and $u_{2} \in K \cap\left(\bar{\Omega}_{R} \backslash \Omega_{r_{2}}\right)$. These are the desired distinct positive solutions of problem (7) for $0<\lambda<\bar{\lambda}_{0}$ and $0<\mu<\bar{\mu}_{0}$ satisfying (18). Then, proof of Part (iii) is complete.

\section{An Example}

To illustrate how our main results can be used in practice, we present an example.
Example 1. For $p=3 / 2$, consider the following boundary value problem:

$$
\begin{gathered}
-\left(\varphi_{p}\left(u^{\prime}\right)\right)^{\prime}=\lambda(t(1-t))^{-1 / 2}\left(t^{2}+1\right)^{1 / 2} u^{p-1}, \quad t \in J, \\
\left.\Delta u\right|_{t=1 / 2}=\mu I_{1}\left(\frac{1}{2}, u\left(\frac{1}{2}\right)\right), \\
\left.\Delta u^{\prime}\right|_{t=1 / 2}=0, \\
u(0)-2 u^{\prime}(0)=\int_{0}^{1} \frac{1}{2} u(t) d t, \quad u^{\prime}(1)=0 .
\end{gathered}
$$

Evidently, $u(t) \equiv 0$ is the trivial solution of problem (83).

\section{Conclusion}

Problem (83) has at least one positive solution for any $\lambda>$ $\sqrt{6} / 4 \pi$ and $\mu>3$.

Proof. Problem (83) can be regarded as a problem of the form (7), where $a=1, b=2$, and

$$
\begin{gathered}
\omega(t)=(t(1-t))^{-1 / 2}, \quad f(t, u)=\left(t^{2}+1\right)^{1 / 2} u^{p-1}, \\
I_{1}(t, u)=(1+t) u, \quad g(t) \equiv \frac{1}{2}, \quad \forall t \in J .
\end{gathered}
$$

It follows from the definition of $\omega, f$, and $g$ that $\left(H_{1}\right)-$ $\left(H_{4}\right)$ hold, and $\omega(t)$ is singular at $t=0$ and $t=1$. By calculating, we have

$$
\begin{gathered}
\int_{0}^{1} \omega(t) d t=\pi, \quad 1 \leq f_{\infty} \leq f^{\infty} \leq 2, \\
1 \leq I_{\infty} \leq I^{\infty} \leq 2, \\
q=3, \quad \delta=\frac{1}{3}, \quad \sigma=\frac{1}{2}, \quad \sigma_{1}=\frac{1}{4}, \\
\gamma=\pi^{2}, \quad m_{r}=\sqrt{\frac{1}{3} r}, \quad m_{r}^{*}=\frac{1}{3} r, \\
\lambda_{0} \geq \frac{\sqrt{6}}{4 \pi}, \quad \mu_{0} \geq 3,
\end{gathered}
$$

where $r>0$ is a constant.

Hence, by Theorem 1(i), the conclusion follows, and the proof is complete.

\section{Conflict of Interests}

The author declares that there is no conflict of interests regarding the publication of this paper.

\section{Acknowledgments}

This work is sponsored by the Project NSFC (11301178 and 11171032) and the Improving Project of Graduate Education of Beijing Information Science and Technology University (YJT201416). 


\section{References}

[1] V. Lakshmikantham, D. D. Bainov, and P. S. Simeonov, Theory of Impulsive Differential Equations, vol. 6 of Series in Modern Applied Mathematics, World Scientific, Singapore, 1989.

[2] A. M. Samoĭlenko and N. A. Perestyuk, Impulsive Differential Equations, vol. 14 of World Scientific Series on Nonlinear Science. Series A: Monographs and Treatises, World Scientific, Singapore, 1995.

[3] M. Benchohra, J. Henderson, and S. Ntouyas, Impulsive Differential Equations and Inclusions, vol. 2 of Contemporary Mathematics and Its Applications, Hindawi Publishing Corporation, New York, NY, USA, 2006.

[4] R. P. Agarwal and D. O'Regan, "Multiple nonnegative solutions for second order impulsive differential equations," Applied Mathematics and Computation, vol. 114, no. 1, pp. 51-59, 2000.

[5] J. J. Nieto and R. Rodríguez-López, "Boundary value problems for a class of impulsive functional equations," Computers \& Mathematics with Applications, vol. 55, no. 12, pp. 2715-2731, 2008.

[6] X. Zhang and M. Feng, "Transformation techniques and fixed point theories to establish the positive solutions of second order impulsive differential equations," Journal of Computational and Applied Mathematics, vol. 271, pp. 117-129, 2014.

[7] W. Ding and M. Han, "Periodic boundary value problem for the second order impulsive functional differential equations," Applied Mathematics and Computation, vol. 155, no. 3, pp. 709726, 2004.

[8] X. Lin and D. Jiang, "Multiple positive solutions of Dirichlet boundary value problems for second order impulsive differential equations," Journal of Mathematical Analysis and Applications, vol. 321, no. 2, pp. 501-514, 2006.

[9] B. Liu and J. Yu, "Existence of solution of $m$-point boundary value problems of second-order differential systems with impulses," Applied Mathematics and Computation, vol. 125, no. 2-3, pp. 155-175, 2002.

[10] M. Feng and D. Xie, "Multiple positive solutions of multipoint boundary value problem for second-order impulsive differential equations," Journal of Computational and Applied Mathematics, vol. 223, no. 1, pp. 438-448, 2009.

[11] M. Feng, B. Du, and W. Ge, "Impulsive boundary value problems with integral boundary conditions and one-dimensional p-Laplacian," Nonlinear Analysis: Theory, Methods \& Applications, vol. 70, no. 9, pp. 3119-3126, 2009.

[12] M. Feng, X. Li, and C. Xue, "Multiple positive solutions for impulsive singular boundary value problems with integral boundary conditions," International Journal of Open Problems in Computer Science and Mathematics, vol. 2, no. 4, pp. 546-561, 2009.

[13] X. Zhang, M. Feng, and W. Ge, "Existence of solutions of boundary value problems with integral boundary conditions for second-order impulsive integro-differential equations in Banach spaces," Journal of Computational and Applied Mathematics, vol. 233, no. 8, pp. 1915-1926, 2010.

[14] W. Ding and Yu. Wang, "New result for a class of impulsive differential equation with integral boundary conditions," Communications in Nonlinear Science and Numerical Simulation, vol. 18, no. 5, pp. 1095-1105, 2013.

[15] G. Infante, P. Pietramala, and M. Zima, "Positive solutions for a class of nonlocal impulsive BVPs via fixed point index," Topological Methods in Nonlinear Analysis, vol. 36, no. 2, pp. 263-284, 2010.
[16] T. Jankowski, "Positive solutions for second order impulsive differential equations involving Stieltjes integral conditions," Nonlinear Analysis: Theory, Methods \& Applications, vol. 74, no. 11, pp. 3775-3785, 2011.

[17] Y. Liu and D. O’Regan, "Multiplicity results using bifurcation techniques for a class of boundary value problems of impulsive differential equations," Communications in Nonlinear Science and Numerical Simulation, vol. 16, no. 4, pp. 1769-1775, 2011.

[18] X. Hao, L. Liu, and Y. Wu, "Positive solutions for second order impulsive differential equations with integral boundary conditions," Communications in Nonlinear Science and Numerical Simulation, vol. 16, no. 1, pp. 101-111, 2011.

[19] J. Sun, H. Chen, and L. Yang, "The existence and multiplicity of solutions for an impulsive differential equation with two parameters via a variational method," Nonlinear Analysis: Theory, Methods \& Applications, vol. 73, no. 2, pp. 440-449, 2010.

[20] J. Shen and W. Wang, "Impulsive boundary value problems with nonlinear boundary conditions," Nonlinear Analysis: Theory, Methods \& Applications, vol. 69, no. 11, pp. 4055-4062, 2008.

[21] L. E. Bobisud, "Steady-state turbulent flow with reaction," The Rocky Mountain Journal of Mathematics, vol. 21, no. 3, pp. 9931007, 1991.

[22] R. Glowinski and J. Rappaz, "Approximation of a nonlinear elliptic problem arising in a non-Newtonian fluid flow model in glaciology," M2AN: Mathematical Modelling and Numerical Analysis, vol. 37, no. 1, pp. 175-186, 2003.

[23] J. Sánchez, "Multiple positive solutions of singular eigenvalue type problems involving the one-dimensional p-Laplacian," Journal of Mathematical Analysis and Applications, vol. 292, no. 2, pp. 401-414, 2004.

[24] G. Dai, R. Ma, and Y. Lu, "Bifurcation from infinity and nodal solutions of quasilinear problems without the signum condition," Journal of Mathematical Analysis and Applications, vol. 397, no. 1, pp. 119-123, 2013.

[25] G. Dai and R. Ma, "Unilateral global bifurcation phenomena and nodal solutions for p-Laplacian," Journal of Differential Equations, vol. 252, no. 3, pp. 2448-2468, 2012.

[26] R. Kajikiya, Y.-H. Lee, and I. Sim, "Bifurcation of sign-changing solutions for one-dimensional $p$-Laplacian with a strong singular weight; $p$-sublinear at $\infty$," Nonlinear Analysis: Theory, Methods \& Applications, vol. 71, no. 3-4, pp. 1235-1249, 2009.

[27] R. Kajikiya, Y.-H. Lee, and I. Sim, "Bifurcation of sign-changing solutions for one-dimensional $p$-Laplacian with a strong singular weight: $p$-superlinear at $\infty$," Nonlinear Analysis: Theory, Methods \& Applications, vol. 74, no. 17, pp. 5833-5843, 2011.

[28] L. Iturriaga and J. Sánchez, "Exact number of solutions of stationary reaction-diffusion equations," Applied Mathematics and Computation, vol. 216, no. 4, pp. 1250-1258, 2010.

[29] D. Ji, W. Ge, and Y. Yang, "The existence of symmetric positive solutions for Sturm-Liouville-like four-point boundary value problem with a $p$-Laplacian operator," Applied Mathematics and Computation, vol. 189, no. 2, pp. 1087-1098, 2007.

[30] R. Kajikiya, Y.-H. Lee, and I. Sim, "One-dimensional pLaplacian with a strong singular indefinite weight. I. Eigenvalue," Journal of Differential Equations, vol. 244, no. 8, pp. 19852019, 2008.

[31] Y. Ding and D. O’Regan, "Positive solutions for a second-order p-Laplacian impulsive boundary value problem," Advances in Difference Equations, vol. 2012, article 159, 2012.

[32] M. Feng, "Multiple positive solutions of fourth-order impulsive differential equations with integral boundary conditions and one-dimensional p-Laplacian," Boundary Value Problems, vol. 2011, Article ID 654871, 26 pages, 2011. 
[33] X. Zhang and W. Ge, "Impulsive boundary value problems involving the one-dimensional p-Laplacian," Nonlinear Analysis: Theory, Methods \& Applications, vol. 70, no. 4, pp. 1692-1701, 2009.

[34] A. Cabada and J. Tomeček, "Extremal solutions for nonlinear functional $\varphi$-Laplacian impulsive equations," Nonlinear Analysis: Theory, Methods \& Applications, vol. 67, no. 3, pp. 827-841, 2007.

[35] J. Henderson and H. Wang, "Nonlinear eigenvalue problems for quasilinear systems," Computers \& Mathematics with Applications, vol. 49, no. 11-12, pp. 1941-1949, 2005.

[36] Z. Yang and D. O'Regan, "Positive solutions of a focal problem for one-dimensional $p$-Laplacian equations," Mathematical and Computer Modelling, vol. 55, no. 7-8, pp. 1942-1950, 2012.

[37] H. Lü, D. O'Regan, and C. Zhong, "Multiple positive solutions for the one-dimensional singular $p$-Laplacian," Applied Mathematics and Computation, vol. 133, no. 2-3, pp. 407-422, 2002.

[38] R. P. Agarwal, H. Lü, and D. O’Regan, "Eigenvalues and the onedimensional p-Laplacian," Journal of Mathematical Analysis and Applications, vol. 266, no. 2, pp. 383-400, 2002.

[39] H. Wang, "On the number of positive solutions of nonlinear systems," Journal of Mathematical Analysis and Applications, vol. 281, no. 1, pp. 287-306, 2003.

[40] G. Bonanno and R. Livrea, "Multiplicity theorems for the Dirichlet problem involving the $p$-Laplacian," Nonlinear Analysis: Theory, Methods \& Applications, vol. 54, no. 1, pp. 1-7, 2003.

[41] Z. Du, X. Lin, and C. C. Tisdell, "A multiplicity result for $p$ Lapacian boundary value problems via critical points theorem," Applied Mathematics and Computation, vol. 205, no. 1, pp. 231237,2008

[42] D.-X. Ma, Z.-J. Du, and W.-G. Ge, "Existence and iteration of monotone positive solutions for multipoint boundary value problem with $p$-Laplacian operator," Computers \& Mathematics with Applications, vol. 50, no. 5-6, pp. 729-739, 2005.

[43] L. Bai and B. Dai, "Three solutions for a $p$-Laplacian boundary value problem with impulsive effects," Applied Mathematics and Computation, vol. 217, no. 24, pp. 9895-9904, 2011.

[44] J. Xu, P. Kang, and Z. Wei, "Singular multipoint impulsive boundary value problem with $p$-Laplacian operator," Journal of Applied Mathematics and Computing, vol. 30, no. 1-2, pp. 105120, 2009.

[45] P. Ning, Q. Huan, and W. Ding, "Existence result for impulsive differential equations with integral boundary conditions," Abstract and Applied Analysis, vol. 2013, Article ID 134691, 9 pages, 2013.

[46] X. Zhang and M. Feng, "Existence of a positive solution for one-dimensional singular $p$-Laplacian problems and its parameter dependence," Journal of Mathematical Analysis and Applications, vol. 413, no. 2, pp. 566-582, 2014.

[47] J. Yan, "Existence of positive periodic solutions of impulsive functional differential equations with two parameters," Journal of Mathematical Analysis and Applications, vol. 327, no. 2, pp. 854-868, 2007.

[48] J. R. Graef, L. Kong, and H. Wang, "Existence, multiplicity, and dependence on a parameter for a periodic boundary value problem," Journal of Differential Equations, vol. 245, no. 5, pp. 1185-1197, 2008.

[49] D. J. Guo and V. Lakshmikantham, Nonlinear Problems in Abstract Cones, vol. 5 of Notes and Reports in Mathematics in Science and Engineering, Academic Press, Boston, Mass, USA, 1988. 


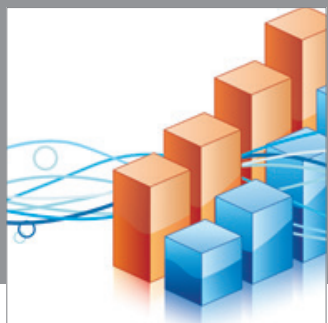

Advances in

Operations Research

mansans

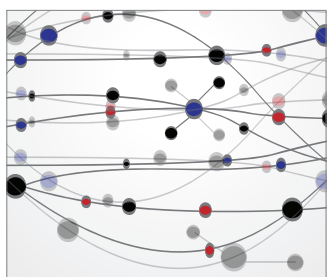

The Scientific World Journal
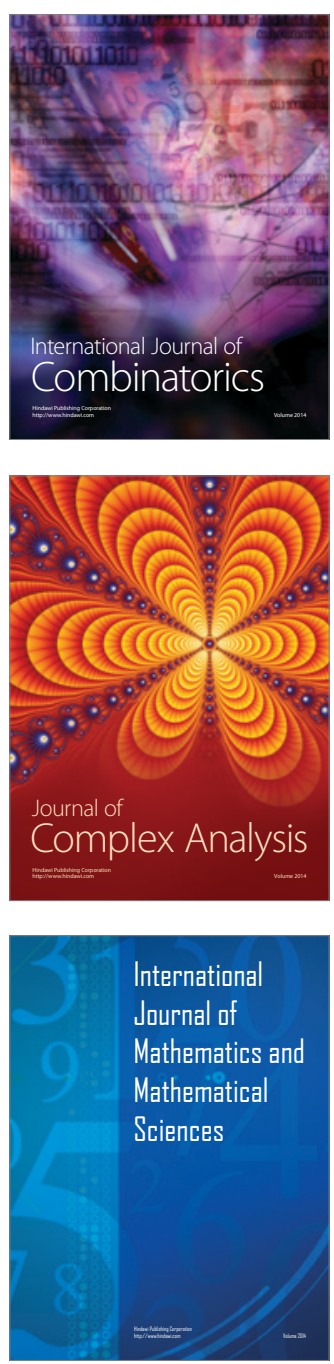
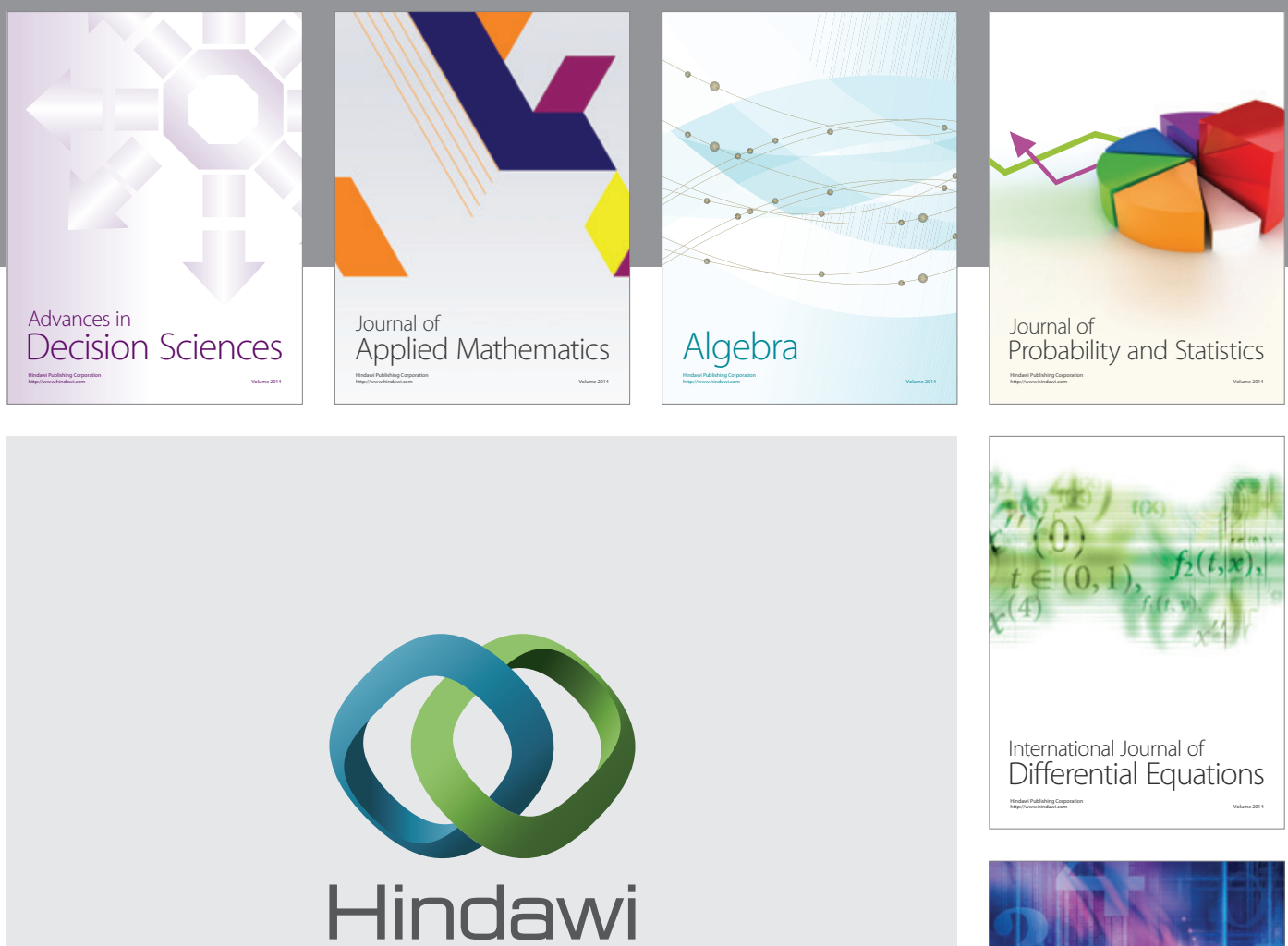

Submit your manuscripts at http://www.hindawi.com
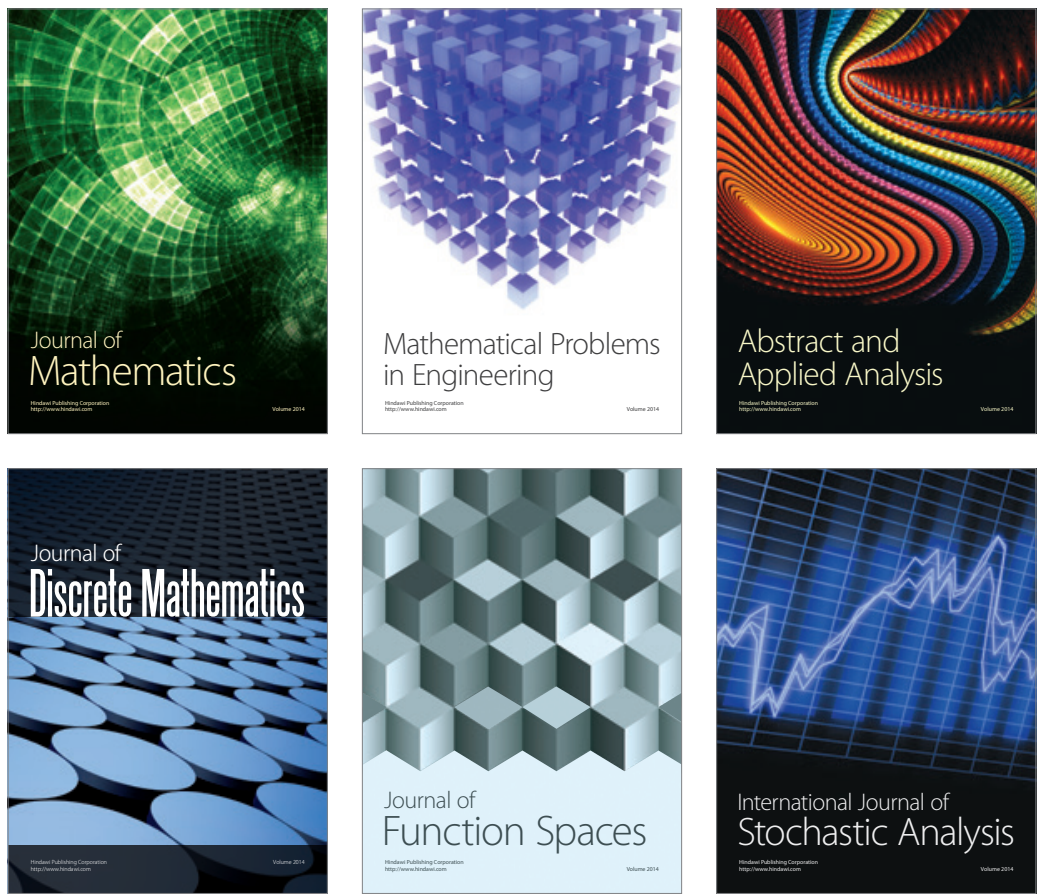

Journal of

Function Spaces

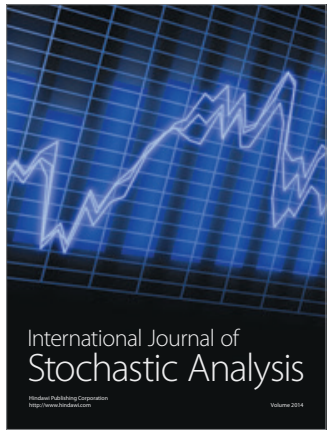

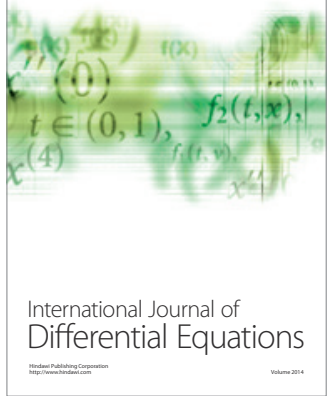
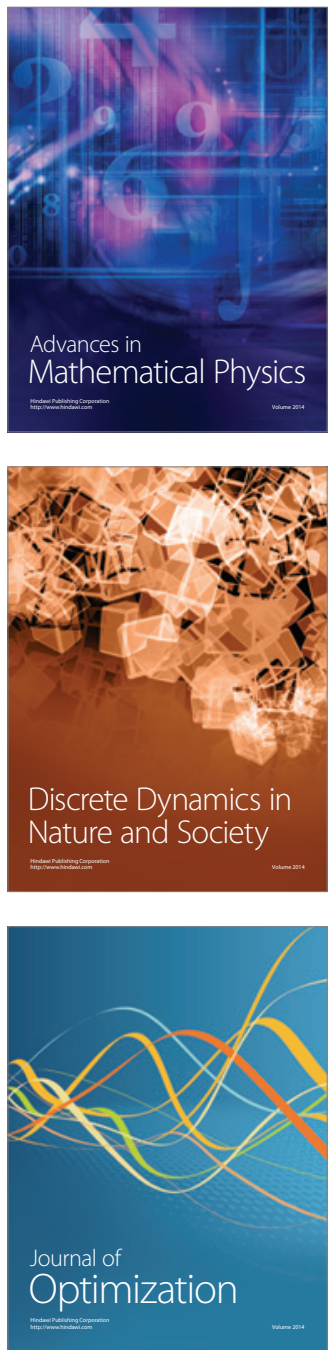